\title{
Applicability of GWTG Score to Heart Failure Patients in South India
}

\author{
Vijay Kumar Bodicherla ${ }^{1} \quad$ Kalyan Chakravarthy ${ }^{1}$ Hemalatha Yellapragada ${ }^{1}$ \\ ${ }^{1}$ Department of Cardiology, Nizams Institute of Medical Sciences, \\ Address for correspondence Vijay Kumar Bodicherla, MD, DM, \\ Hyderabad, Telangana, India \\ Department of Cardiology, Nizams Institute of Medical Sciences, \\ Hyderabad, Telangana, India (e-mail: vijay.bodicherla@gmail.com).
}

Ind J Car Dis Wom 2021;6:8-14.

\begin{abstract}
Keywords

- acute heart failure

- GWTG

- heart failure

- South India

Background The get with the guidelines (GWTG) risk score was developed to predict in-hospital mortality in acute heart failure patients. We aimed to clarify the prognostic impacts of the GWTG risk score in the south Indian heart failure patients admitted to intensive cardiac care unit (ICCU) in our hospital.

Aim Our primary aim was to see the applicability of predicted GWTG risk score of heart failure in the south Indian heart failure patients admitted to intensive cardiac care unit (ICCU) of our hospital. Our secondary aim was to see the event rates and correlate predicted GWTG risk score of heart failure with in-hospital complications.

Materials and Methods We included all the patients admitted to ICCU with the diagnosis of either ischemic or dilated cardiomyopathy over 6 months (January 2018 to June 2018). Indication for admission was either symptomatic heart failure (HF) or to evaluate cause for heart failure. We recorded the demographic and clinical parameters along with the ECG, 2D echo features, and relevant laboratory investigations. The GWTG risk score was based on seven parameters. Race, age, systolic blood pressure, heart rate, blood urea nitrogen (BUN) level, sodium concentration, and presence of chronic obstructive pulmonary disease (COPD) were used to predict in-hospital all-cause mortality, and in-hospital complications were noted.

Results Out of 130 patients, 97 patients fulfilled the inclusion criteria. Out of them, 65 were males, with most of the patients between 40 to 80 years of age. Half the patients were diabetic and had abnormal electrocardiogram (ECG), and more than half were hypertensive, had clinically left ventricular failure (LVF) and diagnosed with ischemic cardiomyopathy (ICMP). Very few were smokers, and $<30 \%$ were alcoholics, had abnormal liver function tests (LFT) and diagnosed with dilated cardiomyopathy (DCMP). Patients were divided into ICMP and DCMP patients, and all the variables were compared. Low systolic blood pressure (BP), abnormal ECG and the mean of GWTG score were greater in 22 patients afflicted with DCMP. However, abnormal renal functions with anemia and more NT-pro-brain natriuretic peptide (NT-proBNP) elevations were observed in ICMP HF patients. Out of 97 patients 70\% patients had GTWG score of 34 to 50 . There were no patients with score $>58$ in our study. Eighteen patients showed in-hospital complications. Five patients died with mean GWTG score 45.00, and they experienced cardiogenic shock with tachycardia and severe LV dysfunction (ejection
\end{abstract}

published online April 14, 2021
DOI https://doi.org/ 10.1055/s-0041-1723918. (c) 2021. Women in Cardiology and Related Sciences.

This is an open access article published by Thieme under the terms of the Creative Commons Attribution-NonDerivative-NonCommercial-License, permitting copying and reproduction so long as the original work is given appropriate credit. Contents may not be used for commercial purposes, or adapted, remixed, transformed or built upon. (https://creativecommons.org/licenses/by-nc-nd/4.0/).

Thieme Medical and Scientific Publishers Pvt. Ltd. A-12, 2nd Floor, Sector 2, Noida-201301 UP, India 
fraction $[\mathrm{EF}]<15 \%$ ), renal failure, hyponatremia, NT-proBNP levels $>25,000 \mathrm{pg} / \mathrm{dl}$ and hepatic derangement at admission. A total of 92 patients were discharged with 39.02 mean GWTG score. There was a significant difference between the mean GWTG scores of patients in their final status (discharged/death) $(p=0.040)$. Also, patients with in-hospital complications had higher GWTG values (on an average 7 scores higher) than without complications $(p=0.000)$.

Conclusions GWTG scores were able to predict (with statistical significance) the true end results for both complications during hospitalization and final discharge/death in hospitalized Indian HF patients. Higher GWTG Scores were an indication of complications or death (39 for complications and 45 for death seems to be the possible average values).

\section{Abstract Image}

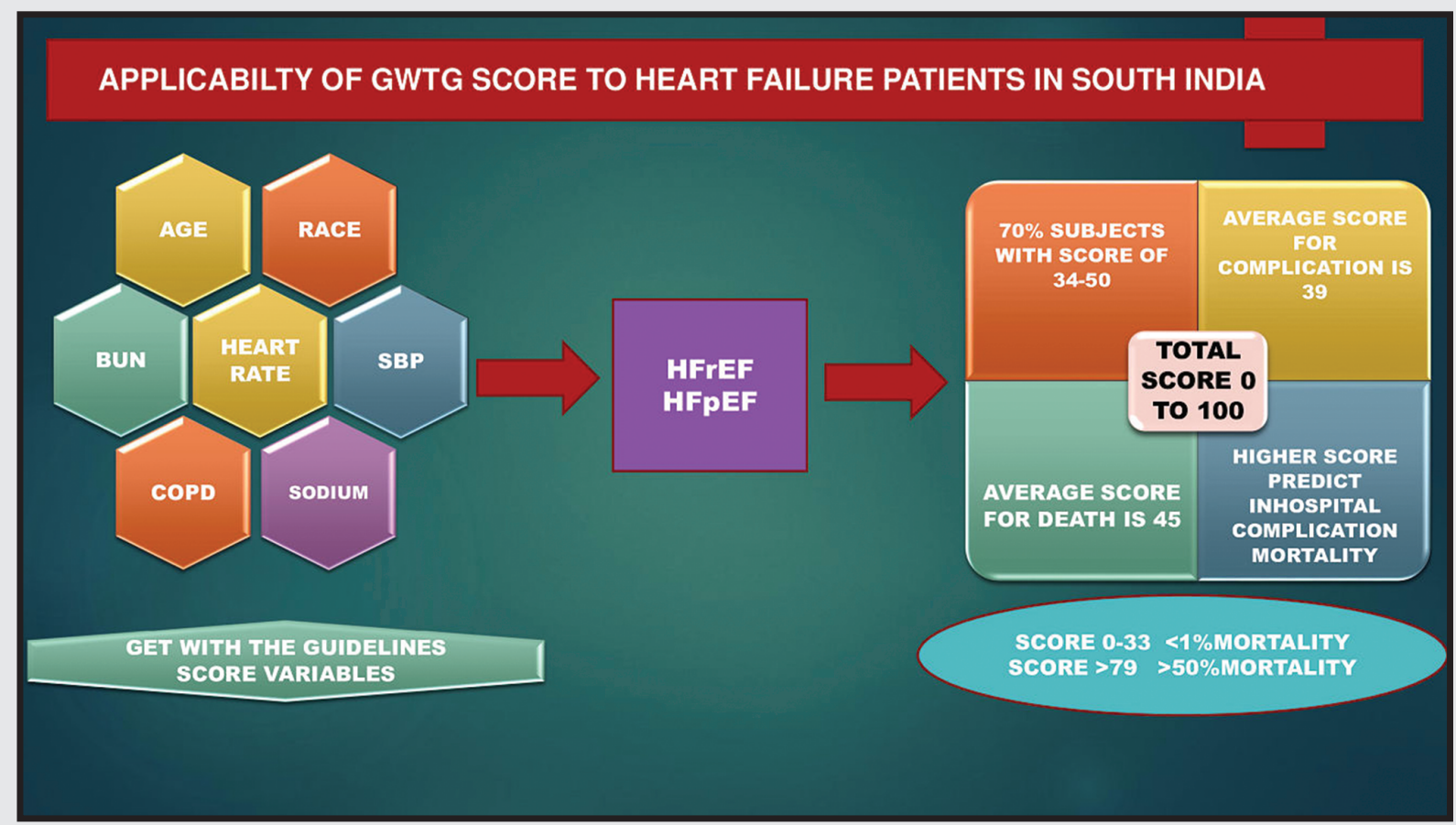

\section{Introduction}

Heart failure (HF) is one of the most common leading causes of death, and it also has high morbidity, which is a high burden to the health care system. ${ }^{1}$ Because of limited resources, risk stratification is necessary in HF patients, which will not only allow us to differentiate between high-risk and low-risk patients but also select appropriate treatment. There are several scores based on multiple parameters now available to predict mortality and cardiovascular events in patients with HF. One of the scores is get with the guidelines (GWTG) risk score, which was established by Peterson et al. ${ }^{2}$ It is basically established from a cohort study which has a role in predicting the prognosis of acute HF and also in chronic phase after discharge.
There is sparse data available, especially from south India regarding utility of this GWTG score. So, our aim is to see the applicability of predicted GWTG risk score of HF among south Indian heart failure patients admitted to the intensive cardiac care (ICCU) of our hospital.

\section{Aim}

Our primary aim is to see the applicability of predicted GWTG risk score of HF among south Indian heart failure patients admitted to the ICCU of our hospital. The secondary aim is to see the event rates and correlate predicted GWTG risk score of heart failure with in-hospital complications. 


\section{Materials and Methods}

We included all the patients admitted to the ICCU with the diagnosis of either ischemic or dilated cardiomyopathy (ICMP or DCMP) over 6 months (January 2018 to June 2018). Indication for admission was either symptomatic HF or to evaluate cause for HF. Patients of HF due to organic valvular heart diseases or due to pulmonary arterial hypertension (primary or secondary) were excluded. We recorded the demographic and clinical parameters along with the ECG, 2D echo features, and relevant laboratory investigations. In-hospital complications like recurrence or worsening of $\mathrm{HF}$, requirement of ventilation, later development of cardiogenic shock or pericardial effusion, and death were noted.

\section{Results}

A total of 130 patients with HF were admitted to the ICCU of our unit during the study period, of which 97 patients fulfilled the inclusion criteria. These 97 patients had either ICMP or DCMP. Out of them, 65 were males ( - Fig. 1), with most of the patients between 40 to 80 years of age ( - Table $\mathbf{1}$ ).

The history of the patients showed half the patients were diabetic and had abnormal electrocardiogram (ECG), and more than half were hypertensive, had clinically left ventricular failure (LVF) and diagnosed with ICMP. Very few were smokers, and $<30 \%$ were alcohol consumers, had abnormal liver function tests (LFT) and diagnosed with DCMP (-Table 2). In - Table 3, clinical and laboratory parameters of the patients were mentioned.

The population was divided into ICMP patients and DCMP patients and all the variables were compared (-Table 4). The average of systolic BP (SBP), pulse and the mean of GWTG score were greater in 22 patients afflicted with DCMP. There was no difference in age and ejection fraction (EF) between the two groups. As expected, coronary artery disease (CAD) risk factors were more frequent in ICMP patients who were statistically significant. DCMP patients were more tachycardic with low SBP, abnormal ECG, and more GWTG score.

\section{TOTAL PATIENTS}

\section{$\square$ males $\square$ females}

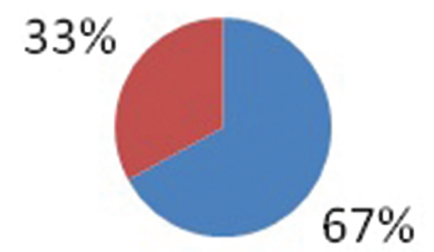

Fig. 1 Pie diagram showing sex distribution in study population.
Table 1 Age distribution of study population

\begin{tabular}{|l|l|l|l|}
\hline Ages & Males & Females & Percentage \\
\hline $10-40$ & 4 & 3 & $6 \%$ \\
\hline $40-80$ & 59 & 28 & $91 \%$ \\
\hline $80-100$ & 2 & 1 & $3 \%$ \\
\hline
\end{tabular}

Table 2 Demographic features of study patients

\begin{tabular}{|l|l|l|}
\hline Demographics parameters & $\begin{array}{l}\text { No. of } \\
\text { patients }\end{array}$ & Percentage \\
\hline DM & 56 & $58 \%$ \\
\hline HTN & 62 & $64 \%$ \\
\hline Smokers & 16 & $16 \%$ \\
\hline Alcoholics & 26 & $27 \%$ \\
\hline Abnormal ECG & 45 & $46 \%$ \\
\hline Clinically LVF & 75 & $77 \%$ \\
\hline $\begin{array}{l}\text { Diagnosis } \\
\text { ICMP }\end{array}$ & 75 & $77 \%$ \\
\hline DCMP & 22 & $23 \%$ \\
\hline Abnormal LFT & 24 & $25 \%$ \\
\hline
\end{tabular}

Abbreviations: DCMP, dilated cardiomyopathy; DM, diabetic mellitus; ECG, echocardiogram; HTN, hypertension; ICMP, ischemic cardiomyopathy; LFT, liver function test.

Table 3 Showing the clinical and laboratory investigation information on average in the study population

\begin{tabular}{|l|l|}
\hline Parameters & Average \\
\hline Pulse (beats/min) & 95.69 \\
\hline Systolic BP $(\mathrm{mm}$ of $\mathrm{Hg})$ & 116.39 \\
\hline EF $(\%)$ & 20.47 \\
\hline Urea $(\mathrm{mg} / \mathrm{dl})$ & 50.68 \\
\hline Creatinine $(\mathrm{mg} / \mathrm{dl})$ & 1.97 \\
\hline Sodium $(\mathrm{meq} / \mathrm{dl})$ & 135.69 \\
\hline HB $(\mathrm{gm} / \mathrm{dl})$ & 11.65 \\
\hline NT-ProBNP $(\mathrm{pg} / \mathrm{mL})$ & 20750 \\
\hline
\end{tabular}

Abbreviations: BP, blood pressure; EF, ejection fraction; $\mathrm{HB}$, hemoglobin; NT-ProBNP, NT-pro-brain natriuretic peptide.

However, abnormal renal functions with anemia and more NT-pro-brain natriuretic peptide (NT-proBNP) elevations were observed in ICMP HF patients.

Concomitant diseases were mentioned in - Table 5 . Cardiogenic shock (SBP $<90 \mathrm{~mm}$ of $\mathrm{Hg}$ ), complete heart block (CHB), chronic obstructive pulmonary diseases (COPD), and chronic kidney disease (CKD) were more frequent in ICMP group, whereas atrial fibrillation (AF) and infection precipitating congestive heart failure (CCF) were more with DCMP patients.

GWTG HF risk score helps to predict in-hospital all-cause HF mortality. Major clinical data was used to get the score numbers. These score numbers were divided into groups, and the total population of 97 were placed into these groups as per their scores (-Table 6 ). As much as $70 \%$ of the population was in group 2 . There were no patients with score > 58 in our study. 
Out of 97 HF patients, 18 patients showed in-hospital complications. The details of complication are mentioned in - Table 7. Out of six ventilated patients, five patients died. Even though pericardial effusion may be due to CCF, but it fell under the purview of in-hospital complications, as this was not present, and at the time of admission, it appeared later in the hospital course. In all cases, it was mild effusion only and did not contribute to hemodynamic compromise. Eight patients showed initial stabilization, were shifted to the wards, and required transfer to ICCU due to recurrence of HF in hospital. Cardiogenic shock was there in 8 patients, and 4 patients were in refractory shock even with maximum inotropic support after 48 hours, but none developed shock requiring inotrope support after the admission.

Table 4 Comparison of variables in both groups

\begin{tabular}{|l|l|l|l|}
\hline Variables & DCMP & ICMP & pvalue \\
\hline No. of cases & 22 & 75 & - \\
\hline Average age & 58 & 54.74 & 0.13 \\
\hline DM (no of pts) & 6 & 50 & 0.00001 \\
\hline HTN (no of pts) & 4 & 58 & 0.00001 \\
\hline Smokers (no of pts) & 0 & 16 & - \\
\hline Alcohol (no of pts) & 4 & 22 & 0.00001 \\
\hline Average pulse (beats/min) & 105.64 & 92.77 & 0.0065 \\
\hline $\begin{array}{l}\text { Average systolic BP } \\
\text { (mm of Hg) }\end{array}$ & 104.55 & 119.87 & 0.0038 \\
\hline Abnormal ECG & 16 & 11 & 0.00001 \\
\hline Average EF & 22.04 & 20.01 & 0.29 \\
\hline Average urea (mg/dl) & 45.37 & 52.24 & 0.09 \\
\hline Average creatinine (mg/dl) & 1.16 & 2.21 & 0.017 \\
\hline Average sodium (meq/dl) & 133.1 & 136.21 & 0.02 \\
\hline Average HB (gm/dl) & 12.66 & 11.35 & 0.00004 \\
\hline Average LFT & 8 & 10 & 0.00001 \\
\hline AVG NT-ProBNP (pg/mL) & 16250 & 22171 & 0.0015 \\
\hline Mean GWTG score & 43.73 & 38.04 & 0.00065 \\
\hline Abbreviations: BP, blod presse & DCMP, & & \\
\hline
\end{tabular}

Abbreviations: BP, blood pressure; DCMP, dilated cardiomyopathy; DM, diabetic mellitus; ECG, echocardiogram; EF, ejection fraction; CWTG, get with the guidelines; HB, hemoglobin; HTN, hypertension; ICMP, ischemic cardiomyopathy; LFT, liver function test; NT-ProBNP, NT-pro-brain natriuretic peptide.

Table 5 Concomitant diseases in both groups

\begin{tabular}{|l|l|l|}
\hline Concomitant diagnosis & DCMP & ICMP \\
\hline Infection (pneumonia) & 6 & 3 \\
\hline CHF with cardiogenic shock & 2 & 6 \\
\hline Paroxysmal AF & 4 & 0 \\
\hline CKD & 0 & 6 \\
\hline COPD & 0 & 6 \\
\hline CHB & 0 & 6 \\
\hline AKI & 3 & 6 \\
\hline
\end{tabular}

Abbreviations: AF, atrial fibrillation; AKI, acute kidney injury; $\mathrm{CHB}$, complete heart block; CHF, chronic heart failure; CKD, chronic kidney disease; COPD, chronic obstructive pulmonary disorder; DCMP, dilated cardiomyopathy; ICMP, ischemic cardiomyopathy.
In - Table 8, details of five died patients were given. All five patients admitted experienced cardiogenic shock with tachycardia and severe LV dysfunction (ejection fraction $[\mathrm{EF}]<15 \%$ ), renal failure, hyponatremia, NT-proBNP levels > $25,000 \mathrm{pg} / \mathrm{dl}$ and hepatic derangement.

The important prediction of GWTG risk score is the mortality percentage for hospitalized HF patients. Our primary aim was to correlate the GETG score with actual mortality in our study patients. From the total population of $97 \mathrm{HF}$ patients, five (5\%) deaths occurred with 45.00 mean GWTG and 92 discharged with 39.022 mean GWTG score (-Fig. 2).

From the boxplot, we can say the Q1 of the GWTG score of the death population was 39.00 and Q3 was 50.50, whereas Q1 of the GWTG score of the discharge patients was 34.00 and Q3 was 44.00. The interval plot of the GWTG score has no overlapping, so we can say that there was a significant difference between the mean GWTG scores of patients in their final status (discharged/death $)(p=0.040)$.

Our secondary aim was to compare the GWTG score with in-hospital events even though this score is used only for in-hospital mortality. In - Fig. 3, GWTG scores were compared between patients with in-hospital complication versus those without.

The boxplot of the GWTG score of the 79 patients without in-hospital complications showed a mean score of 38.038, with first quartile (Q1) 33.00 and third quartile 42.00. And the boxplot of the GWTG scores of the 18 patients with

Table 6 GWTG score table

\begin{tabular}{|l|l|l|l|l|}
\hline Group & $\begin{array}{l}\text { Class } \\
\text { of } \\
\text { score }\end{array}$ & $\begin{array}{l}\text { No. of } \\
\text { patients }\end{array}$ & Percentage & $\begin{array}{l}\text { Predicted } \\
\text { mortality } \\
\text { rate }\end{array}$ \\
\hline Group 1 & $0-33$ & 22 & $23 \%$ & $<1 \%$ \\
\hline Group 2 & $34-50$ & 68 & $70 \%$ & $1-5 \%$ \\
\hline Group 3 & $51-57$ & 7 & $7 \%$ & $5-10 \%$ \\
\hline Group 4 & $58-61$ & 0 & $0 \%$ & $10-15 \%$ \\
\hline Group 5 & $62-65$ & 0 & $0 \%$ & $15-20 \%$ \\
\hline Group 6 & $66-70$ & 0 & $0 \%$ & $20-30 \%$ \\
\hline Group 7 & $71-74$ & 0 & $0 \%$ & $30-40 \%$ \\
\hline Group 8 & $75-78$ & 0 & $0 \%$ & $40-50 \%$ \\
\hline Group 9 & $\geq 79$ & 0 & $0 \%$ & $>50 \%$ \\
\hline
\end{tabular}

Abbreviation: GWTG, get with the guidelines.

Table 7 Details of in-hospital complications

\begin{tabular}{|l|l|l|}
\hline Type of event & $\begin{array}{l}\text { No. of } \\
\text { patients }\end{array}$ & Percentage \\
\hline Pericardial effusion & 4 & $4.12 \%$ \\
\hline Recurrence HF & 8 & $8.25 \%$ \\
\hline $\begin{array}{l}\text { Ventilated (including 5 } \\
\text { deaths) }\end{array}$ & 6 & $6.19 \%$ \\
\hline Death & 5 & $5.15 \%$ \\
\hline Total & 18 & $18.56 \%$ \\
\hline
\end{tabular}

Abbreviation: HF, heart failure. 
in-hospital complications show the mean score of 45.00, Q1 39.50 and Q3 51.00. The GWTG score was different in patients with or without in-hospital complications (38 vs. 45 ), which was statistically significant $(p=0.000)$. The interference of

Table 8 Details of death patients

\begin{tabular}{|l|l|}
\hline Variables & Comments \\
\hline No. of patients & 5 \\
\hline Sex & M (2), F (3) \\
\hline DM & None \\
\hline HTN & None \\
\hline Smokers & None \\
\hline Alcoholics & None \\
\hline Average pulse & 112 \\
\hline $\begin{array}{l}\text { Average systolic BP } \\
\text { (mm of Hg) }\end{array}$ & 80 \\
\hline ECG & Abnormal \\
\hline Average EF (\%) & $<15$ \\
\hline Urea mg/dl & 65 \\
\hline Creatinine mg/dl & 2.3 \\
\hline Na meq/l & 129 \\
\hline HB gm/l & 11 \\
\hline LFT & $\begin{array}{l}\text { Abnormal } \\
\text { cardiogenic shock }\end{array}$ \\
\hline NT-ProBNP (pg/dl) & On D5, they were ventilated, then on D8, \\
\hline Diagnosis & 45 \\
\hline In-hospital events & Ihey died \\
\hline Mean GWTG score & A wF with \\
\hline
\end{tabular}

Abbreviations: BP, blood pressure; DM, diabetes mellitus; EF, ejection fraction; GWTG, get with the guidelines; HB, hemoglobin; HTN, hypertension; NT-ProBNP, NT-pro-brain natriuretic peptide.

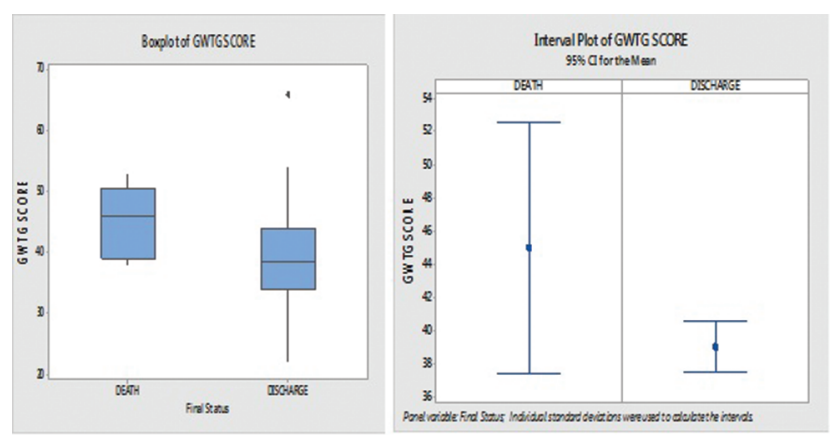

Fig. 2 Box and interval plot of the get with the guidelines (GWTG) score of died and discharged patients.
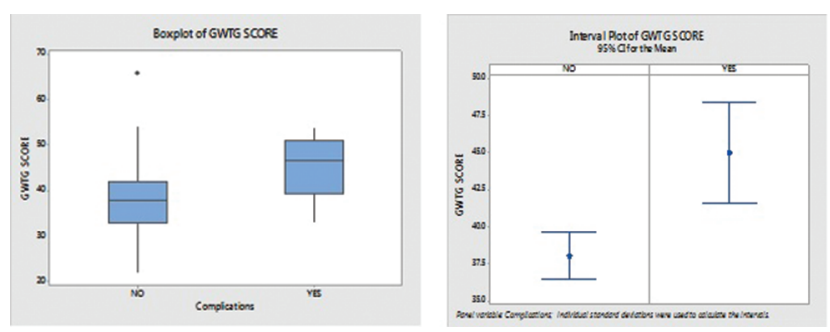

Fig. 3 Box and interval plot of get with the guidelines (GWTG) score of patients with or without in-hospital complications. both the population GWTG scores were different, as patients with in-hospital complications had higher GWTG values (on an average 7 scores higher) than the GWTG score of the patients without $(p=0.000)$.

\section{Discussion}

American Heart Association (AHA) GWTG risk score predicts the mortality in hospital admitted patients with both reduced and preserved LV systolic function. In these patients, GWTG risk score usage is different, appropriate, and easy when compared with other risk stratification methods. The seven variables which are included in GWTG score are routinely collected at the time of admission ${ }^{4}$ Heart rate, nonBlack race, COPD, and serum sodium are independent predictors of mortality, but they were less important with regard to the points contributed by them to the total GWTG HF risk score. LV systolic function which is a risk predictor in some models is not present in this risk score ${ }^{3}$. So, absence of LVEF at time of admission does not limit prognostication; hence, using this model and this risk score has identical characteristics operating in both group of patients, so that this score can be applicable to a broad spectrum of heart failure patients.

As per standard guidelines, we have calculated GWTG score from race, age, SBP, heart rate, blood urea nitrogen (BUN) level, sodium levels, and presence of COPD. Presence of Black race (points 0 or 3 ), COPD ( 0 or 2 ), age between 19 to 110 years (0-28), SBP 50 to 200 (0-28), BUN 9 to $150(0-28)$, heart rate 79 to $105(0-8)$, and serum sodium 130 to 139 (0-4) scoring is given. Then, we have calculated GWTG scores. Older age, low SBP, elevated heart rate, low serum sodium, elevated BUN, presence of COPD, and nonBlack race are predicted to have increased risk of death. ${ }^{4}$ Age, SBP, and BUN contributed more to the overall point score, whereas heart rate, presence of COPD, serum sodium, and nonBlack race contributed relatively few points to the overall score. Subsequently, patients were divided as groups from 1 to 9 , according to the GWTG score (0-79). In our study, most of the patients come under group $2(70 \%, N=68)$ with GWTG score of 34 to 50 (-Table 9).

There are various risk scoring systems available to predict in-hospital mortality, but these available risk stratification models have some limitations.

The Acute Decompensated Heart Failure National Registry (ADHERE) study identified serum creatinine, BUN and SBP as the best predictors of in-hospital mortality using classification and regression tree analysis. ${ }^{5}$ As it uses only three variables, it is appealing to classify patients as low, intermediate, or highrisk. However, it does not include all variables which give more precise characterization of individual risk and significantly inform outcomes. The limitation is it gives overly high mortality of the low-risk group in comparison with other models. Likewise, acute heart failure (AHF) index has same limit.

OPTIMIZE-HF include variables like race, serum creatinine, serum sodium, age, HR, liver disease, previous cerebrovascular accident (CVA)/transient ischemic attack (TIA), peripheral vascular disease, left ventricular systolic dysfunction, COPD, SBP, previous HF hospitalization and limits, in that it does not 
Table 9 GWTG risk score

\begin{tabular}{|c|c|c|c|c|c|c|c|}
\hline Systolic BP & Points & BUN & Points & Sodium & Point & Age & Points \\
\hline $50-59$ & 28 & $<9$ & 0 & $<130$ & 4 & $<19$ & 0 \\
\hline $60-69$ & 26 & $10-19$ & 2 & 131 & 3 & $20-29$ & 3 \\
\hline $70-79$ & 24 & $20-29$ & 4 & 132 & 3 & $30-39$ & 6 \\
\hline $80-89$ & 23 & $30-39$ & 6 & 133 & 3 & $40-49$ & 8 \\
\hline 90-99 & 21 & $40-49$ & 8 & 134 & 2 & $50-59$ & 11 \\
\hline $100-109$ & 19 & $50-59$ & 9 & 135 & 2 & $60-69$ & 14 \\
\hline 110-119 & 17 & $60-69$ & 11 & 136 & 2 & $70-79$ & 17 \\
\hline $120-129$ & 15 & $70-79$ & 13 & 137 & 1 & $80-89$ & 19 \\
\hline 130-139 & 13 & $80-89$ & 15 & 138 & 1 & $90-99$ & 22 \\
\hline $140-149$ & 11 & $96-49$ & 17 & $\geq 139$ & 0 & 100-109 & 25 \\
\hline 150-159 & 9 & $106-109$ & 19 & & & $\geq 110$ & 28 \\
\hline $160-169$ & 8 & $110-119$ & 21 & & & & \\
\hline $170-179$ & 6 & $120-129$ & 23 & & & & \\
\hline $180-189$ & 4 & $130-139$ & 24 & & & & \\
\hline 190-199 & 2 & $140-149$ & 26 & & & & \\
\hline$\geq 200$ & 0 & $\geq 150$ & 28 & & & & \\
\hline Heart beat & Points & Black point & Points & COPD & Points & $\begin{array}{l}\text { Total } \\
\text { score }\end{array}$ & Probability of death \\
\hline$<79$ & 0 & Yes & 0 & Yes & 2 & $0-33$ & $<1 \%$ \\
\hline $80-84$ & 1 & No & 3 & No & 0 & $34-50$ & $1.50 \%$ \\
\hline $85-89$ & 3 & & & & & $51-57$ & $>5-10 \%$ \\
\hline $90-94$ & 4 & & & & & $58-61$ & $>15-20 \%$ \\
\hline $95-99$ & 5 & & & & & $62-65$ & $>20-30 \%$ \\
\hline $100-104$ & 6 & & & & & $66-70$ & $>30-40 \%$ \\
\hline \multirow[t]{2}{*}{$\geq 105$} & 7 & & & & & $71-74$ & $>40-50 \%$ \\
\hline & & & & & & $75-78$ & $>50 \%$ \\
\hline
\end{tabular}

Abbreviations: BP, blood pressure; BUN, blood urea nitrogen; COPD, chronic obstructive pulmonary disorder.

include data on BUN at admission and does not have a separate derivation and validation cohort. ${ }^{6}$

The international "placebo-controlled randomized study of the selective A1 adenosine receptor antagonist rolofylline for patients hospitalized with acute decompensated heart failure and volume overload to assess treatment effect on congestion and renal function" (PROTECT) trial enrolled 2033 patients hospitalized with AHF and with mild-to-moderate renal function impairment. The limitation of this trial is that it did not include patients taking inotropic agents, patients with preserved $\mathrm{EF}$, recent $\mathrm{MI}$, and those with severe pulmonary disease; therefore, so the fact that it cannot be applicable to a wide range of community-based populations is its limitation. ${ }^{7}$

Enhanced Feedback for Effective Cardiac Treatment (EFFECT) and Outcomes of a Prospective Trial of Intravenous Milrinone for Exacerbations of Chronic Heart Failure (OPTIME-CHF) models. However, both these trials were established for 30- and 60-days mortality after hospitalization, and the OPTIME-CHF trial is not applicable to broad spectrum of patients, as it is limited to patients cohorts with LV systolic dysfunction only. Additional predictive models for long-term mortality have been reported but limited by the use of large numbers of variables, derivation in clinical trial populations, and inclusion of variables that are not typically assessed in clinical care, such as uric acid, peak oxygen consumption, or timing of diagnosis of HF., ${ }^{8} 9$

GWTG- HF risk score has several advantages and strengths and it differs from other risk scores, as it is based on patients with diverse demographic variables and wide range of comorbidities. With regard to LV systolic function, as it is applicable for HF with both reduced and preserved $\mathrm{EF}$, it can be widely applicable. Additionally, the variables which were used are small in number and can be collected at the time of admission. Several other models of risk score prediction have more than 20 variables. ${ }^{10}$ They are not 
feasible and difficult to use in routine clinical practice as compared with GWTG risk score.

Hauptman et $\mathrm{al}^{11}$ found that among physicians caring for patients with HF, only a few clinicians can predict death accurately. Generally, physicians substantially overestimate the risk and overutilize the resources in critical care. Conversely, it demonstrates that medical therapy for HF is poorly calibrated to risk, with high-risk patients paradoxically receiving evidence-based medical therapy less often. ${ }^{12}$

Our study results are comparable with previous studies in case of in-hospital mortality. High GWTG score is associated with a greater death rate. Our study revealed that high GWTG score is also associated with a high incidence of in-hospital events.

Thus GWTG HF risk score can be used for patient risk quantification, thus facilitating patient triage and encouraging the use of evidence-based therapy in high-risk patients, so that therapy can efficiently be used for high-risk patients by reducing utilization in low-risk patients.

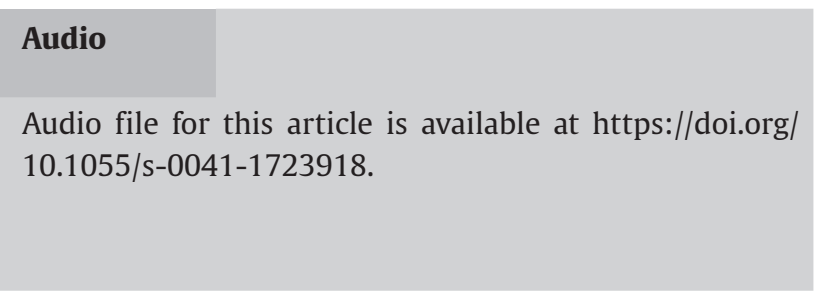

\section{References}

1 Ambrosy AP, Fonarow GC, Butler J, et al. The global health and economic burden of hospitalizations for heart failure: lessons learned from hospitalized heart failure registries. J Am Coll Cardiol 2014;63:1123-1133
2 Peterson PN, Rumsfeld JS, Liang L, et al; American Heart Association Get With the Guidelines-Heart Failure Program. A validated risk score for in-hospital mortality in patients with heart failure from the American Heart Association Get With the Guidelines program. Circ Cardiovasc Qual Outcomes 2010;3:25-32

3 Pocock SJ, Wang D, Pfeffer MA, et al CHARM Investigators, on behalf of the. Predictors of mortality and morbidity in patients with chronic heart failure. Eur Heart J 2006;27:65-75

4 Suzuki S, Yoshihisa A, Sato Y, et al. Clinical significance of get with the guidelines-heart failure risk score in patients with chronic heart failure after hospitalization. J Am Heart Assoc 2018;7:e008316

5 Fonarow GC, Adams KF Jr, Abraham WT, Yancy CW, Boscardin WJ. For the ADHERE Scientific Advisory Committee SGaI. Risk stratification for in-hospital mortality in acutely decompensated heart failure: classification and regression tree analysis. JAMA 2005;293:572-580

6 Abraham WT, Fonarow GC, Albert NM, et al. Predictors of in-hospital mortality in patients hospitalized for heart failure: insights from the Organized Program to Initiate Lifesaving Treatment in Hospitalized Patients With Heart Failure (OPTIMIZE-HF). J Am Coll Cardiol 2008;52:347-356

7 O'Connor CM, Mentz RJ, Cotter G, Metra M, Cleland JG, Davison BA. The PROTECT In-Hospital Risk Model: 7-day outcome in patients hospitalized with acute heart failure and renal dysfunction. Heart Fail 2012;14(6):605-612

8 Felker GM, Leimberger JD, Califf RM, et al. Risk stratification after hospitalization for decompensated heart failure. J Card Fail 2004;10:460-466

9 Lee DS, Austin PC, Rouleau JL, Liu PP, Naimark D, Tu JV. Predicting mortality among patients hospitalized for heart failure: derivation and validation of a clinical model. JAMA 2003;290:2581-2587

10 Levy WC, Mozaffarian D, Linker DT, et al. The Seattle Heart Failure Model: prediction of survival in heart failure. Circulation 2006;113:1424-1433

11 Hauptman PJ, Swindle J, Hussain Z, Biener L, Burroughs TE. Physician attitudes toward end-stage heart failure: a national survey. Am J Med 2008;121:127-135

12 Lee DS, Tu JV, Juurlink DN, et al. Risk-treatment mismatch in the pharmacotherapy of heart failure. JAMA 2005;294:1240-1247 